\title{
Parkinsonian tremor loses its alternating aspect during non-REM sleep and is inhibited by REM sleep
}

\author{
J J M Askenasy, M D Yahr
}

\begin{abstract}
Non-REM sleep transforms the waking alternating Parkinsonian tremor into subclinical repetitive muscle contractions whose amplitude and duration decrease as non-REM sleep progresses from stages I to IV. During REM sleep Parkinsonian tremor disappears while the isolated muscle events increase significantly.
\end{abstract}

Changes in serotoninergic, aminergic, cholinergic and neuropeptidergic neurotransmitters occur in sleep. ${ }^{12}$ These changes influence the basal ganglia and locus niger ${ }^{3}$ (fig 1).

By activating the hypnagogic structures sleep may influence the Parkinsonian features. Gowers commented on "the persistence of tremor during the dormant state". ${ }^{4}$ At the turn of the century Froment showed that the disappearance of Parkinsonian tremor during sleep is a constant feature. ${ }^{5}$ In 1964 Brain described it as an important diagnostic feature of Parkinson's disease. ${ }^{6}$ The introduction of polysomnography led a number of authors to study the persistence of the tremor during sleep. ${ }^{7-10}$

In his experiments Jouvet disturbed the REM sleep in cats by depleting the dopaminergic vesicles with reserpine, and restoring REM sleep with dopamine precursors. ${ }^{11}$ Recently, we showed reversal of the sleep disturbance in Parkinson's disease by dopaminergic agonists, demonstrating that biogenic amines have a role in sleep. ${ }^{12} 13$

We aimed to study the behaviour of the dynamic changes of Parkinsonian tremor during sleep.

Department of

Neurology, Sleep

Research Institute,

Sackler School of

Medicine, Tel-Aviv

University, Tel-Aviv,

Israel

J J M Askenasy

Department of

Neurology, Mount

Sinai School of

Medicine, New York

University, New York,

United States

M D Yahr

Correspondence to:

J J M Askenasy,

Sleep Research Institute,

Sheba Medical Center

Tel-Hashomer 52621, Israel.

Received 11 October 1988

and in final revised form

14 November 1989 .

Accepted 21 December 1989 physiological and intention tremor were present. Their treatment was reduced to a standard of $125 / 500 \mathrm{mg}$ carbidopa/levodopa. A depressive patient, treated with the monoamine oxidase inhibitor (MAOI) phenelzine, $45 \mathrm{mg}$ per day, was added to this study. Ten normal subjects matched for sex and age constituted the control group.

Two techniques were used in parallel:

1) Polysomnography for two nights following an adaptation night, was scored according to the standards of Rechtschaffen and Kales. ${ }^{14}$ Polysomnography consisted of: electroencephalography recorded with gold cup electrodes at C3-A1/A2 with a DC resistance less than 3000 Ohms. Electro-oculography (EOG) was recorded with Ag-AgCl Beckman electrodes which were located lateral to the right and left outer canthi. Electromyography (EMG) was recorded with Beckman surface electrodes interspaced at $15 \mathrm{~mm}$ and applied as close as possible to the motor point of the muscle surface. The EMG band pass was adjusted to $1-100 \mathrm{~Hz}$ with a $10 \times$ lower sensitivity. The time constants were 0.003 for EMG, 0.3 for EEG and 5 for EOG. The

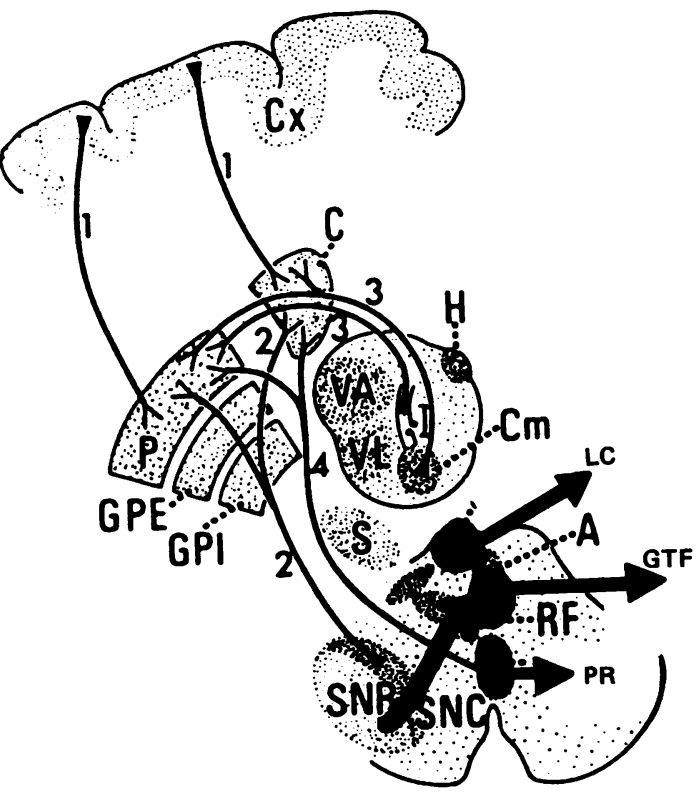

Figure 1 Interrelations between sleep and extrapyramidal structures. $C=$ caudate; $P=$ putamen extrapyramidal structures. $C=$ caudate; $P$
$S N R=$ pars reticularis of substantia nigra;

$S N R=$ pars reticularis of substantia nigra; $S N C=$ pars compacta of substantia nigra; $L C=$ locus caeruleus; $G T F=$ gigantocellular tegmental field; $P R=$ pontine raphe nuclei. 


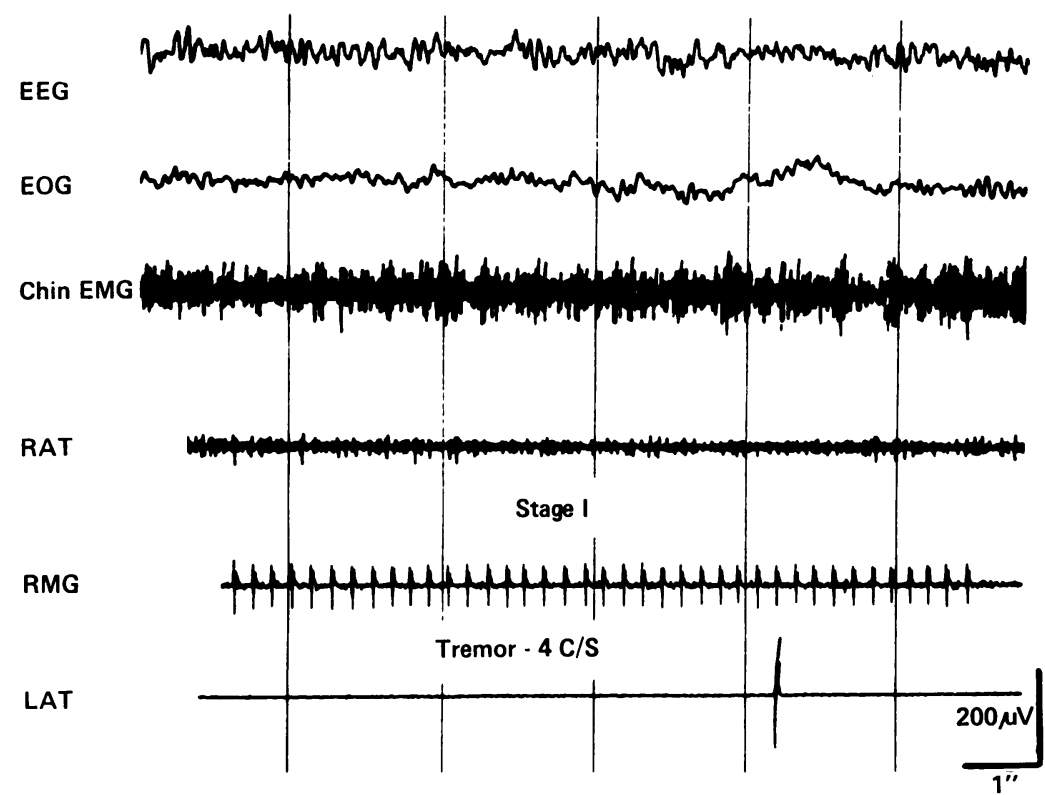

Figure 2 Repetitive muscle contractions in non-REM sleep stage I. RAT = right anterior tibial EMG;RMG = right medial gastrocnemius $E M G ; L A T=$ left anterior tibial EMG. recorded muscles for the upper limbs were flexor carpi ulnaris and extensor digitorum and for the lower limbs anterior tibialis and medial gastrocnemius. Respiration and electrocardiography were recorded by means of transducers and surface electrodes. A video-tape closed-circuit television ensured a continuous videomonitoring.

Muscle events were scored separately for each epoch of 30 seconds on specially designed files. Transition epochs and epochs including arousals less that $1 / 4$, as well as transition epochs were included.

2) The second technique consisted of a multichannel recording system of the anterior tibialis by means of 16 surface electrodes monitoring muscle potentials on eight channels.

For scoring tremor the following criteria were used:

a) Tremor was defined based on the repetitiveness of the time interval between multiple EMG potentials only, without

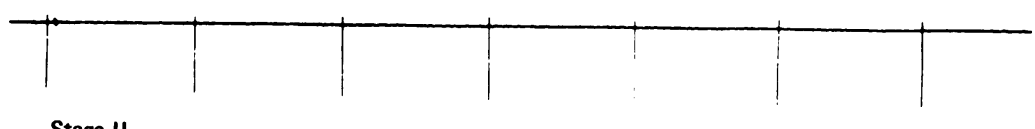

Stage II

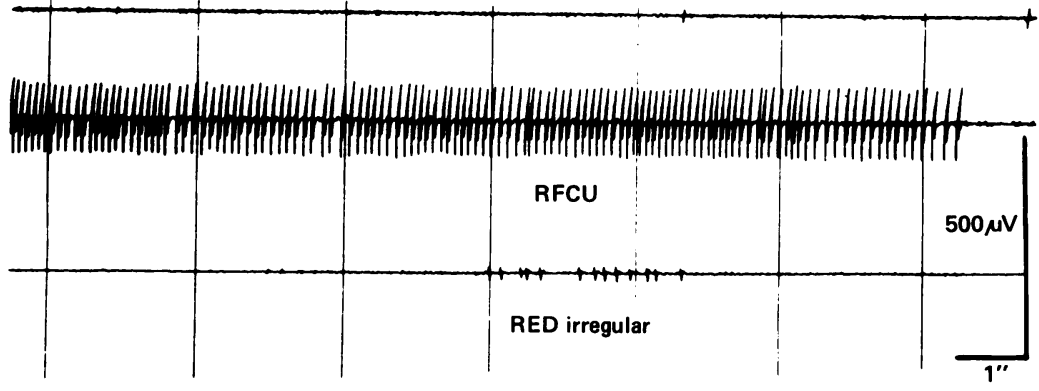

Figure 3 Repetitive muscle contractions in non-REM sleep stage II. RFCU = right flexor carpi ulnaris EMG; RED = right extensor digitorum EMG. reference to amplitude; b) One bout of tremor was scored as one muscle event when rhythmic repetitive multiple potentials appeared for at least five equal periods; c) Burst-tremor was defined as a short-lasting interference pattern followed by repetitive EMG potentials of equal time intervals. The burst-tremors were scored as bursts and not as tremors.

All the EMG events were scored per sleep stage for every sleep cycle separately on five specially designed files. Standard deviations and ANOVA tests of significance were computed.

\section{Results}

The scoring of the sleep patterns in the Parkinson's disease patients, stages III-IV, showed the presence of a light fragmented sleep when compared with the normal controls. The PD patients slept $62 \%$ of the time compared with $82 \%$ in the controls. The mean number of arousals per total sleep time was 12 compared with four in the controls.

Parkinsonian tremor was present during the non-REM sleep in all sleep stages in the 10 Parkinsonian patients. Parkinsonian tremor was absent during REM sleep in all 10 Parkinsonian patients (fig 2,3 ). The rhythmic repetitive multiple potentials of one episode of tremor displayed moderate variations of the beat to beat amplitude. In the MAOI-treated patient tremor was also present during all non-REM sleep stages.

In the two severe tremor subgroups tremor was quantitatively increased compared with the two non-tremor subgroups, $p<0.05$. Despite being statistically non-significant there was more tremor during sleep in the severe tremor-severe rigidity subgroup than the severe tremor-mild rigidity subgroup.

Tremor during sleep was predominant in the muscles which showed the most tremor during wakefulness.

Analysis of the inter-relationship between video-monitored movement and EMG tremor showed that Parkinsonian tremor during sleep was not associated with movement. Parkinsonian tremor during sleep is a subclinical event. Twitches associated with bursts appeared in sleep stage II and sleep stage I, but not in REM sleep. The EMG bursts did not have clinical correlates when they were shorter than $600 \mathrm{~ms}$, and their amplitude was less than $175 \mathrm{mv}$.

The Parkinsonian tremor during sleep lacks the alternating aspect and consists of repetitive muscle contractions of the agonists, antagonists and synergists. During the short arousals $(<3 \mathrm{~s})$ between sleep periods the alternating aspect of wake tremor is sometimes lacking (fig 4).

Three major variables of the Parkinsonian tremor during sleep were analysed: frequency, amplitude, and duration. All the frequencies of repetitive muscle contractions from 1 to $13 \mathrm{~Hz}$ were found during sleep in Parkinsonian patients. But in each individual in the same muscle for the same sleep stage, the frequency 


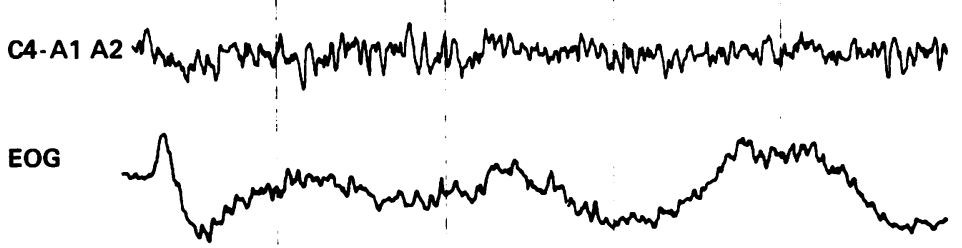

Chin EMG

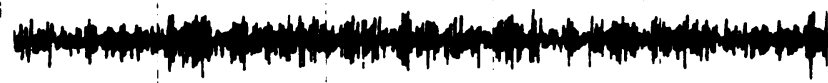

Stage W

RAT II C/S

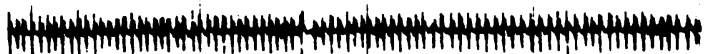

RMG $4 \mathrm{C} / \mathrm{S}$

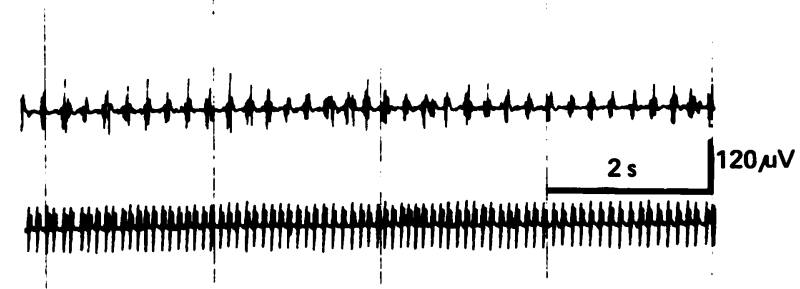

Figure 4 Short arousal during sleep stage II showing repetitive muscle contractions in 3 different rates of frequency. $C_{4}-A_{1} A_{2}=$ electroencephalogram;

$E O G=$ electrooculogram; The last four channels display the electromyograms of the chin; $R A T=$ right anterior tibial; $R M G=$ right medial gastrocnemius acid;

$L A T=$ left anterior tibial.
REM sleep abolishes repetitive muscle contractions. The continuous random muscle phasic events during REM sleep which are not associated with movement are considerably increased in Parkinsonian patients. When quantifying the sleep electromyographic events (EMGE) in PD patients, we found that the mean (SD) number of EMGE per minute of sleep was $2 \cdot 81(1 \cdot 15)$ in the lower limbs compared with $1.25(0.91)$ in the controls and 1.36 $(0.94)$ in upper limbs compared with 0.79 $(0.32)$ for the controls.

The temporal distribution of muscle phasic events behave in a contradictory way to repetitive muscle contractions during REM sleep (fig 5 ).

During the arousals between sleep, episodes of tremor were sometimes associated with movement which persisted during sleep Stage I but disappeared with the onset of sleep Stage II. The bursts of motor unit potentials, usually associated with twitch movements (category 1 ) in Parkinsonian patients appear as bursttremor (fig 6).

The burst-tremor events appeared in sleep Stages I and II, but of significantly high frequency in sleep Stage II ( $p<0.05)$. Among the normal controls, a 51 year old man showed four episodes of repetitive muscle contractions with a frequency of $4 \mathrm{c} / \mathrm{s}$ during sleep.

was constant or changed less than $1 \mathrm{~Hz}$ towards the end of the sleep stage.

There was a direct relationship between the frequency of the sleep repetitive muscle contractions and the tonic muscle activity of the muscle. Flexors exhibited a more increased tonic muscle activity and more repetitive muscle contractions than extensors during sleep.

Amplitude decreases linearly with the nonREM sleep progression from Stage I to IV and parallels the decrease in tonic muscle activity.

The mean duration of repetitive muscle contractions was $30 \mathrm{~s}$ with a range of $6-48 \mathrm{~s}$. The repetitive muscle contraction duration became more and more fragmented with non-REM sleep progression from Stage I to IV.

Amplitude and duration of repetitive muscle contractions may decrease to a tenth of their initial value with the sleep cycle progression from Stage I to IV.

Figure 5 The dissociation of repetitive muscle contractions and EMGE during REM sleep. The mean of muscle events and tremor bouts of two nights in one patient.

$E M G E=$ electro-

myographic events. $W=$ wakefulness.

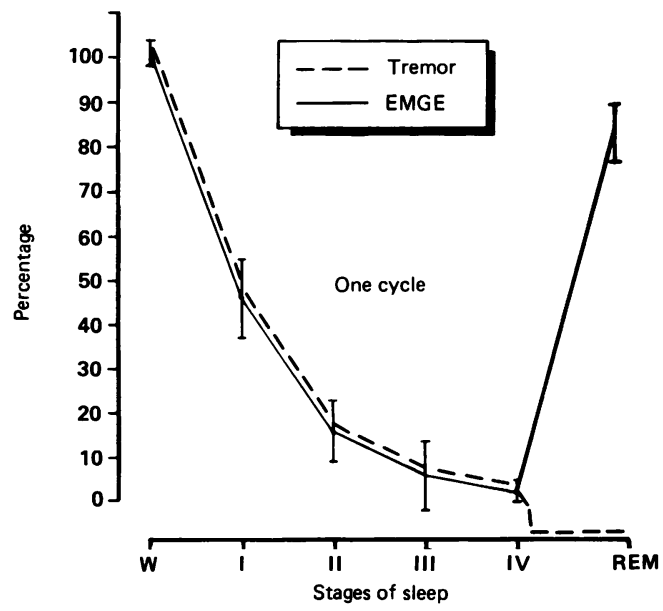

\section{Discussion}

The resting tremor of 4-6 Hz during wakefulness was found in $25^{\circ}$ of the Parkinsonian patients. Resting tremor may be associated with action tremor, enhanced physiological tremor, or essential tremor. ${ }^{15-19}$ In the Parkinsonian resting tremor, the alternating aspect is a constant waking characteristic, ${ }^{15} 16$ while essential and postural tremor was shown to be either "alternating" or "synchronous". ${ }^{1516}$ Our study shows that the alternating aspect of Parkinsonian tremor disappears during sleep, and flexors and extensors oscillate independently with non-alternating frequencies. Interestingly this characteristic was present in familial tremor during wakefulness. ${ }^{19}$ It seems that the alternating aspect of Parkinsonian tremor is conditioned by an aroused nervous system. Two phenomena could be associated with the disappearance of alternating Parkinsonian tremor during sleep: a) The existence of a suppressive phenomenon ensuring inactive periods of $200 \mathrm{~ms}$. In theory this suppressive phenomenon originated in the nucleus ventralis intermedius (VIM), rhythmic firing of which exhibits the frequency of the tremor. ${ }^{20} 21$ VIM destruction abolishes tremor during wakefulness. ${ }^{20} \mathrm{We}$ are not aware of any other studies on muscle activity during sleep in stereotactic VIM lesioned patients. It may be hypothesised that the very strong inhibition of VIM activity during paradoxical sleep may explain the inhibition of repetitive muscle contractions during REM sleep. Moreover, even in patients treated with MAOI whose REM sleep is characterised by a motor phasic hyperactivity, REM sleep suppresses the repetitive muscle contractions. ${ }^{22}$ 
EEG

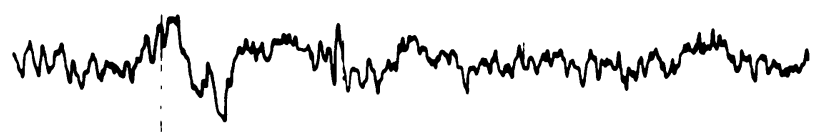

EOG

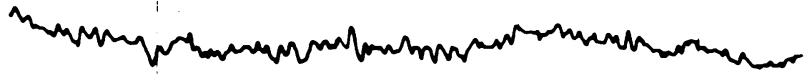

Chin EMG

RAT

RMG

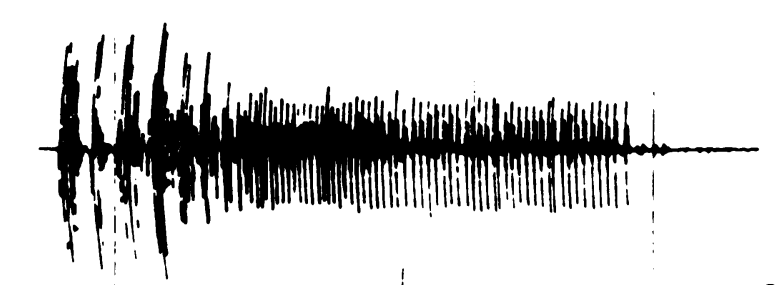

$200 \mu \mathrm{V}$

Stage II

Figure 6 Burst-tremor during sleep stage II in PD patients.

b) The alternating tremor was considered to be the consequence of a reciprocal inhibition of the agonists while antagonists contract and vice-versa. ${ }^{2324}$ During sleep there is a lack of reciprocal inhibition, which may contribute to the transformation of the alternating tremor into repetitive muscle contractions. ${ }^{26}$ Denny Brown believed that reciprocal inhibition is lacking when the basal ganglia are damaged. ${ }^{23} 24$

During wakefulness the frequency of the alternating tremor depends on the grade of muscle tone. ${ }^{25}$ This direct relationship persists and is not influenced by the sleep process. The fact that during sleep the subgroup of patients with severe tremor-severe rigidity is more tremorigenic than the subgroup of severe tremor-mild rigidity, supports this observation.

Two mechanisms could underly the selfmaintained Parkinsonian tremor during wakefulness: central and peripheral. ${ }^{2627}$ According to this concept duration and amplitude seem to have a strong cortical component, and frequency mainly a subcortical and peripheral one. $^{2627}$

Frequency is the most stable feature of the repetitive muscle contraction during sleep in Parkinsonian patients. The dramatic change of the amplitude and duration suggests that the two variables are strongly influenced by the dynamics of the sleep process, while the pacemakers responsible for the repetitive muscle contraction frequency are not.

This study reports a complete absence of tremor during REM sleep contrasting with the significant increase of EMG isolated motor muscle potentials. ${ }^{1012132229}$ While Parkinsonian tremor parallels the EMG events during the non-REM sleep, dissociation appears during REM sleep with the disappearance of tremor. It seems that the isolated motor muscle potentials escape to the strong postsynaptic

inhibition characterising REM sleep. ${ }^{28} 29$ These results contradict previous observations. ${ }^{930}$

In summary, the sleep process strongly influences the Parkinsonian feature of tremor. Unalternating tremor persists during all stages of non-REM sleep at a subclinical level and disappears during REM sleep. The alternating aspect of Parkinsonian tremor characterises wakefulness and is lacking during sleep in which tremor is transformed into repetitive muscle contractions. Parkinson's intuition in his book published in 1817, at a time when very little was known about sleep, presented two situations in which the sleep-wake tremor contrast was evident: ${ }^{31}$ "The tremulous motion of the limbs occur during sleep, and augment until they awaken the patient" (p7). "His attendants (case VI) observed that of late the trembling would sometimes begin in his sleep, and increase until it awakened him"'(p17).

1 Moruzzi G. The historical development of the deafferentation hypothesis of sleep. Proc Am Phil Soc 1964;108:19-28

2 Jouvet $M$. Recherches sur les structures nerveuses et les mechanismes responsables des differentes phases du sommeil physiologique. Arch Ital Biol 1962;100:125-206.

3 Hobson JA. The reciprocal interaction model of sleep cycle control. Implication for PGO wave generation and dream control. Implication for $P G O$ wave generation and dream amnesia. In: Drucker-Collin R, McGaugh J, eds.
Neurobiology of Sleep and Memory. New York: Academic Neurobiology of Sleep and
Press Inc, 1977:159-83.

4 Gowers WR. A manual of diseases of the nervous system. Philadelphia: Blakiston, 1901.

5 Froment J, Delore P. Le tremblement Parkinsonien n'est pas un tremblement de repos. Rev Neurol 1926;1:46-51.

6 Brain WR. Clinical neurology, 2nd ed. London: Oxford University Press, 1964:67.

7 Tassinari CA, Broughton R, Poire R, Roger J, Gastaut H. Sur l'evolution des mouvements anormaux au cours du sommeil. In: Fishgold $\mathrm{H}$, ed. Le sommeil de nuit normal et pathologique. Paris: Masson et Cie, 1965.

8 April R. Observations on Parkinsonian tremor in all night sleep. Neurology 1966;16:72-4.

9 Mano T, Schiozawa Z, Sobue Y. Polygraphic study of extrapyramidal involuntary movements in man during sleep. Proc Third Internat Cong Sleep Research, Tokyo; 1979:220.

10 Askenasy JJM. Sleep patterns in extrapyamidal disorders. Internat J Neurol 1981;15:62-76.

11 Jouvet $M$. Biogenic amines and the states of sleep. Science 1969;163:32-41.

12 Askenasy JJM, Yahr MD. Reversal of sleep disturbance in Parkinson's disease by anti-Parkinsonian therapy: a preliminary study. Neurology 1985;35:527-32.

13 Askenasy JJM, Weitzman ED, Yahr MD. Are periodic movements in sleep a basal ganglia dysfunction? J Neural Transm 1985;70:337-47.

14 Rechschaffen A, Kales A. A manual of standardised terminology, techniques and scoring system for sleep stages of human subjects. Washington, DC: Public Health Service, US Government Printing Office, 1968.

15 Andrews CG, Burke D, Lance JW. The comparison of tremors in normal, Parkinsonian and athetotic man. tremors in normal, Parkin
$J$ Neurol Sci 1973;19:53-61.

16 Shahani BT, Young RR. Physiological and pharmacological aids in the differential diagnosis of tremor. $J$ Neurol Neurosurg Psychiatry 1976;39:772-83.

17 Growdon JH, Young RR, Shahani BT. The differential diagnosis of tremor in Parkinson's disease. Trans Am Neurol Assoc 1975;100:37-9.

18 Young RR. Enhanced physiological tremor in Parkinson's Disease. In: Yahr MD, ed. Current concepts of Parkinson's disease and related disorders. Amsterdam: Excerpta Medica, 1983:56-72.

19 Elbe RJ. Physiologic and essential tremor. Neurology 1986;36:225-31.

20 Narabayashi H, Ohye C. Pathological tremor: Its generating mechanism and treatment. In: Yahr MD, ed. Current concepts of Parkinson's disease and related disorders. Amsterdam: Excerpta Medica, 1983:47-55.

21 Narabayashi H. Clinical analysis of akinesia. J Neurol Trans 1988;16 (suppl):129-36.

22 Askenasy JJM, Yahr MD. Is monoamine oxidase inhibitor induced myoclonus serotoninergically mediated? J Neural Trans 1988;72:67-76. 
23 Denny-Brown $\mathrm{D}$. The basal ganglia and their relation to disorders of movement. London: Oxford University Press, 1962.

24 Denny-Brown D. Clinical symptomatology of diseases of basal ganglia. In: Vinken PJ, Bruyn GW, eds. Handbook of clinical neurology, Vol 6. diseases of basal ganglia. North Holland; Amsterdam, 1978:133-72.

25 Gresty MA, Findley LJ. Tremors in Parkinson's disease. In: Rose FC Capildeo $R$ eds. Research progression Parkinson's disease. Wells, Pitman Medical Lrogression. 75-87.

26 Rondot P, Bathien N. Tremor: Clinical Physiological Studies. In: Yahr MD, ed. Current concepts of Parkinson's disease and related disorders. Amsterdam: Excerpta Medica, 1983:17-30.
27 Rondot P, Bathien N. Pathophysiology of Parkinsonian tremor. In: Desmedt JE, ed. Progress in clinical neurophysiology Vol 5. Basel, Karger 1978:138-49.

28 Askenasy JJM, Yahr MD, Davidovitch S. Isolated phasic discharges in anterior tiberal muscle: a stable feature of paradoxical sleep. J Clin Neurophysiol 1988;5:175-81.

29 Askenasy JJM, Yahr MD. Correlation of sleep patterns with muscle activity in patients with Parkinson's disease. In: Yahr MD, ed. Current concepts of Parkinson's disease and related disorders. Amsterdam: Excerpta Medica, 1983: 172-94.

30 Stern M, Roffwarg H, Duvoisin R. The Parkinsonian tremor in sleep. J Nerv Men Dis 1968;147:202-10.

31 Parkinson J. Essay on the Shaking Palsy, London: Sherwood Nealy and Jones, 1817. 